\title{
Dust in Hot Environments: Giant Dusty Galactic Halos
}

\author{
Andrea Ferrara
}

Osservatorio Astrofisico di Arcetri, Largo Fermi 5, 50125 Firenze, Italy

\begin{abstract}
I review some of the evidences for dust in the Local Bubble and in galactic halos and show that a general mechanism based on radiation pressure is capable of evacuating dust grains from regions dominated by massive star energy input and thus originate huge dusty halos. A Monte Carlo/particle model has been developed to study the dust dynamics above HII chimneys and the results, among other findings, show that dust can travel several kpc away from the plane of the parent galaxy. The cosmological implications of extragalactic dust are briefly outlined.
\end{abstract}

\section{Introduction}

The evolution of dust in hot environments is a very important topic in astrophysics with a variety of implications. There are several excellent reviews on the subject (Draine \& Salpeter 1979, Seab 1987, McKee 1989, Dwek \& Arendt 1992) - and in particular on the issue of grain survival - to which I defer the interested reader for a complete treatment. Instead, in the spirit of a highlight talk, I would like to focus on and isolate one particular effect, namely the dynamics of grains in a hot environment, and show how this might affect the structure and evolution of a galaxy and perhaps even our understanding of the distant universe, as other speakers at this Colloquium have emphasized (see Finkbeiner's contribution).

In this sense, three "hot" environments - the Local Bubble (LB), Galactic Halos and the Intergalactic Medium (IGM) - central to the subject of this Colloquium are probably different aspects of the same phenomenon: the feedback of star formation and supernovae on the interstellar medium of a galaxy, whose manifestation is the disk/halo interaction. The central point of this paper is that the disk/halo interaction - and in particular its dusty form - is indeed crucial to understand several galactic properties and also the distant universe. The relatively well studied local environment might then represent the Rosetta stone to interpret the interstellar processes occurring during the epoch of galaxy formation. 


\section{Dust in the Local Bubble}

Contrary to what one might expect, not much is known yet about dust in the LB. The most firm results come from a pioneering paper by Tinbergen (1982). This author observed that stars closer than $\simeq 35 \mathrm{pc}$ from the sun in the direction of the Galactic Center show appreciable polarization (polarization degree of the order of $0.02 \%$ ). From the same data Frisch (1995) found no correlation between the polarization degree and the the distance to the sample stars. This could indicate that the dust is closer than the nearest star, and therefore located in the neutral cloud embedding the sun, commonly named the Local Fluff. One might also be led to speculate that the amount of dust outside this very small region of a few parsecs size is very limited. Another interesting feature is the absence of polarization in the anticenter hemisphere, which could be explained by a lack of grain alignment and/or a different configuration of the magnetic field. It has to be pointed out, though, that Leroy (1993) did a very similar study to the one of Tinbergen, failing to confirm the above discussed polarization; however, this could be accounted for by the lower sensitivity of Leroy's data. New promising perspectives for the investigation of dust in the local environment are opened by ongoing infrared emission studies (Reach, this conference) and in-situ experiments (Landgraf, this conference).

The absence of polarization outside the very local region might have different interpretations. If one is inclined to attribute this effect to a real deficiency of grains rather than to other reasons, such as a poor alignment, then the issue of grain destruction or evacuation should be addressed. Grains in a hot gas are essentially destroyed via thermal sputtering, i.e. collisions with ions or electrons with Maxwellian velocity distribution. The hot gas in the LB is now thought to have temperature $\sim 10^{6} \mathrm{~K}$ and number density $n \sim 5 \times 10^{-3} \mathrm{~cm}^{-3}$. For these figures, the sputtering time calculated using the recent sputtering yields by Tielens et al. (1994), for a $0.01 \mu \mathrm{m}$ grain is $\sim 10^{8} \mathrm{yr}$, much longer than the estimated age of the LB. Dust destruction can have been caused by the supernova shock that has generated the hot bubble, whose mechanical luminosity has been inferred to be equal to $\sim 2.8 \times 10^{36} \mathrm{ergs} \mathrm{s}^{-1}$ by Frisch (1995). However, in spite of the still poorly understood underlying physics, it seems unlikely that the efficiency of grain destruction in a shock can be higher that $10 \%$ (McKee 1989). Grains are more likely to be destroyed behind radiative shocks, by the combined effects of a greatly enhanced gas density and betatron acceleration that increases the grain Larmor frequency. There is clear evidence of supersonic motions in cloudlets inside the Local Fluff, and collisions among them necessarily produce shocks. Ricotti, Ferrara \& Miniati (1997) have shown that supersonic cloud-cloud collisions are inelastic for a wide range of parameters, which, stated in an alternate manner, implies that the corresponding shocks are radiative; this result has now been confirmed by extensive hydro and MHD numerical simulations performed by Miniati et al. (1997). Thus, the presence of dust in a $5 \mathrm{pc}$ sphere around the sun, 
confirms that the dust destruction has not been very efficient. This forms a basis for our hypothesis that dust can have been evacuated from the LB rather than destroyed locally. The LB is an example, and probably far cry from being the most spectacular one (see Normandeau et al. 1996), of the effects of (multi)-supernova explosions resulting in the vertical tunnelling of hot gas inside HI walls - in brief a galactic "chimney". It is reasonable to expect that the global effects of a collection of such objects might profoundly influence the structure and properties of the ISM of galaxies and of their halos. A very brief summary of some observational evidence that might hint at the relevance of supernova energy injection in shaping the dust distribution in galaxies is given in the next Section.

\section{Observational Facts on Dust Large-Scale Distribution}

The tremendous observational improvement occurred in the last few years has substantially challenged some of our long-standing prejudices about the distribution of dust in galaxies. Dust has long been believed to be confined in a very thin disk, with approximately the same horizontal extension as disk stars. However, this does not appear to be the case from the first dedicated observations which tackle the problem exploiting the range of wavelengths appropriate for the detection of cold dust, i.e. FIR and sub-mm bands. Davies et al. (1997), from an analysis of $140 \mu \mathrm{m}$ and $240 \mu \mathrm{m}$ DIRBE observations of the Galaxy demonstrated very convincingly the existence of an extended (scale height $\sim 0.5 \mathrm{kpc}$ ), cool $(T \sim 18-22 \mathrm{~K})$ dust component. They favour the interpretation that "...this dust may be supported high above the plane by radiation pressure". Dust therefore seems to be present in the lower halo of the Milky Way at least at the same distance as the HI gas in the Lockman layer. Sofue et al. 1994, pointed out the presence of a variety of dust structures (arcs, loops, bubbles, streamers) in the halo of NGC 253 extending into the first $3 \mathrm{kpc}$ of the halo of that galaxies. This limit is merely being set by instrumental sensitivity. This adds to the already well established evidences of similar phenomena in several edge-on galaxies (where the identification is definitely easier); for a review see Dettmar (1992). Not only is the vertical distribution in spirals more extented than previously thought, but similar conclusions are emerging also for the one in the disk plane. Observations of NGC 6949 in the ISO $200 \mu \mathrm{m}$ band have shown that a cold dust component exists which is considerably more extended compared to that measured by IRAS in the same galaxy and sampling a warmer component. Zaritsky (1994), studying the B and I colors of distant galaxies seen through the halo of two nearby spirals, concluded that background galaxies at smaller projected separations are statistically redder than those in the outer regions. This fact suggests the existence of an extended dust halo with a derived scale length of $31 \pm 8 \mathrm{kpc}$. This figure is in remarkable agreement with the prediction of 
Heisler \& Ostriker (1988), who required a scale length of $33 \mathrm{kpc}$ to account for the observed quasar number counts.

\section{Grain Dynamics Above Bubbles}

There are two main mechanisms able to evacuate dust from the interior of a supernova driven interstellar bubble and inject it into the halo: (i) a convective flow (i.e. a "galactic fountain", Shapiro \& Field [1976] or a chimney, Norman \& Ikeuchi [1989]) in which the gas heated by a supernova blast wave becomes buoyant and raises into the halo carrying along the dust grains that are coupled with the gas; (ii) a wind driven by the radiation pressure above the clusters of young OB stars. In order to work efficiently, the first mechanism requires the occurrence of the so-called "blow-out" in which the gas of the shell created by the explosion is effectively reaccelerated and injected into the halo. However, recent studies have demonstrated that this phenomenon, at least in the Galaxy, should be much rarer than previously thought, either because the growth of the shell is inhibited by the presence of a magnetic field (Tomisaka 1990; MacLow \& Norman 1992) or by effects related to non-coeval star formation (Shull \& Saken 1995), which essentially result in a time dependent mechanical luminosity, in turn producing lower expansion velocities. Here we explore the second possibility, i.e. dust is injected into the intergalactic space by radiation pressure. It is interesting to note that through this mechanism most of the dust is evacuated from the region surrounding the star cluster prior to supernova explosions. As the latter take place, only some dust will be still close enough to the center of the star cluster to be reached and processed by the expanding shock. Thus, at least part of the grain population will have a higher chance of survival. This effect will occur no matter how big the association is (and also for an isolated supernova) since grains will be always pre-exposed to the radiative flux of the stars eventually turning into supernovae.

To substantiate the above points and to understand the radiation-driven dynamics of grains above these so-called "HII chimneys" - vertical, density bounded ionization structures (Dove \& Shull 1994) allowing Lyc photons to escape the disk and penetrate into the halo - we have performed a set of numerical simulations of the above "dusty chimneys" based on a mixed Monte Carlo/particle approach. A complete description of the calculation and of the detailed results will be presented in Ferrara \& Shull (1997). Here we present the main features of the model and discuss some results relevant to the present topic.

We assume that dust grains are immersed in the time-dependent radiation field of a stellar association containing $N$ OB stars (typically $N=100$ ). The spectrum of the radiation field has been adapted from the results of Sutherland \& Shull (private communication) who calculate the evolution of the composite radiation spectrum from the most updated stellar models. 


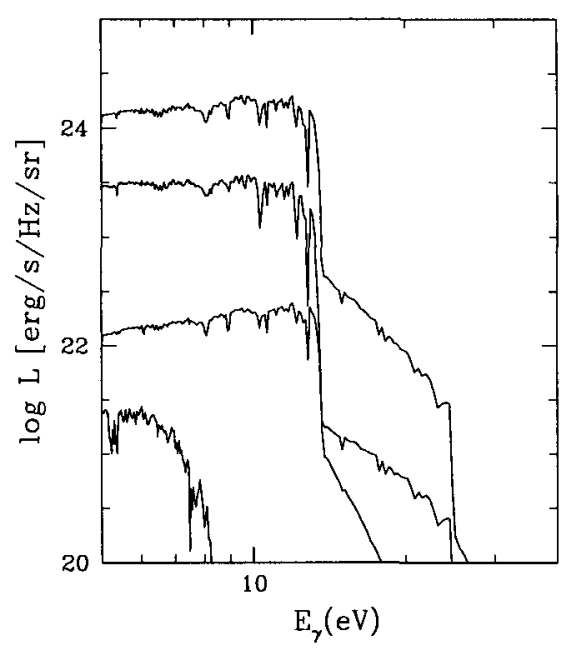

Fig. 1. Time evolution of the radiation spectrum above an OB association containing $N=40 \mathrm{OB}$ stars as a function of photon energy. From top to bottom the curves refer to the evolutionary times $17,29,1,42 \mathrm{Myr}$, respectively.

The evolution of the radiation spectrum for a poor association with $N=40$ is shown in Fig. 1. The luminosity increases up to a maximum and then decreases and fades away as the stars evolve into supernovae. At $t=42 \mathrm{Myr}$ time, the luminosity, $L_{\nu}$, of the radiation field at $5 \mathrm{eV}$ is roughly 3 orders of magnitude lower than the maximum intensity $L_{\nu} \sim 10^{24} \mathrm{erg} \mathrm{s}^{-1} \mathrm{~Hz}^{-1} \mathrm{sr}^{-1}$, reached at $t \sim 17$ Myr. The spectrum is characterized by a Lyc break of about 1.5 decades, and by a flat distribution below the Lyman limit. Our simulations end at $t \sim 50 \mathrm{Myr}$, when there is essentially no power in the radiations to drive the grains.

We take the vertical distribution of the gas, $n_{g}(z)$, from Dickey \& Lockman 1990; the dust distribution, $n_{d}(z)$, is assumed to be initially exponential with a scale height $z_{d}=120 \mathrm{pc}$. The initial position and radius of each (spherical) grain are extracted via a Monte Carlo procedure from the parent distributions, $n_{d}(z)$ and MRN, respectively. We then follow the dynamical evolution of the grain ensemble as driven by radiation, gravity and drag (viscous + coulomb) forces; the grain charge is also calculated consistently solving the detailed balance equation in which both collisional and photoelectric charging rates are included. The grain charge $Z$ is given by the solution of

$$
n_{p}\left\{\left(\frac{8 k T_{e}}{\pi m_{p}}\right)^{1 / 2}\left[\tilde{J}_{\nu_{p}, \tau_{p}}\left(a, Z, T_{e}\right) s_{p}+\delta_{p}\left(a, Z, T_{e}\right)\right]+\tilde{J}_{p e}\left(a, \mathcal{F}_{\nu}\right)\right\}=
$$




$$
n_{e}\left\{\left(\frac{8 k T_{e}}{\pi m_{e}}\right)^{1 / 2}\left[\tilde{J}_{\nu_{e}, \tau_{e}}\left(a, Z, T_{e}\right) s_{e}-\delta_{e}\left(a, Z, T_{e}\right)\right]\right\},
$$

where the index $p, e$ stands for protons and electrons, respectively; $\tilde{J}_{\nu, \tau}$ are the collisional charging rates; $\delta$ are the secondary emission rates; $\tilde{J}_{p e}$ is the photoelectric charging rate; $s$ is the sticking probability. $\tilde{J}_{\nu, \tau}$ are taken from Draine \& Sutin (1987); $\tilde{J}_{p e}$ is taken from Draine (1978); $\delta$ and $s$ are taken from Draine \& Salpeter (1979). The detailed balance equation above must be solved together with the field emission condition (Draine \& Sutin 1987) that limits the value of the charge:

$$
-1-0.7\left(\frac{a}{\mathrm{~nm}}\right)^{2}<Z<1+21\left(\frac{a}{\mathrm{~nm}}\right)^{2}
$$
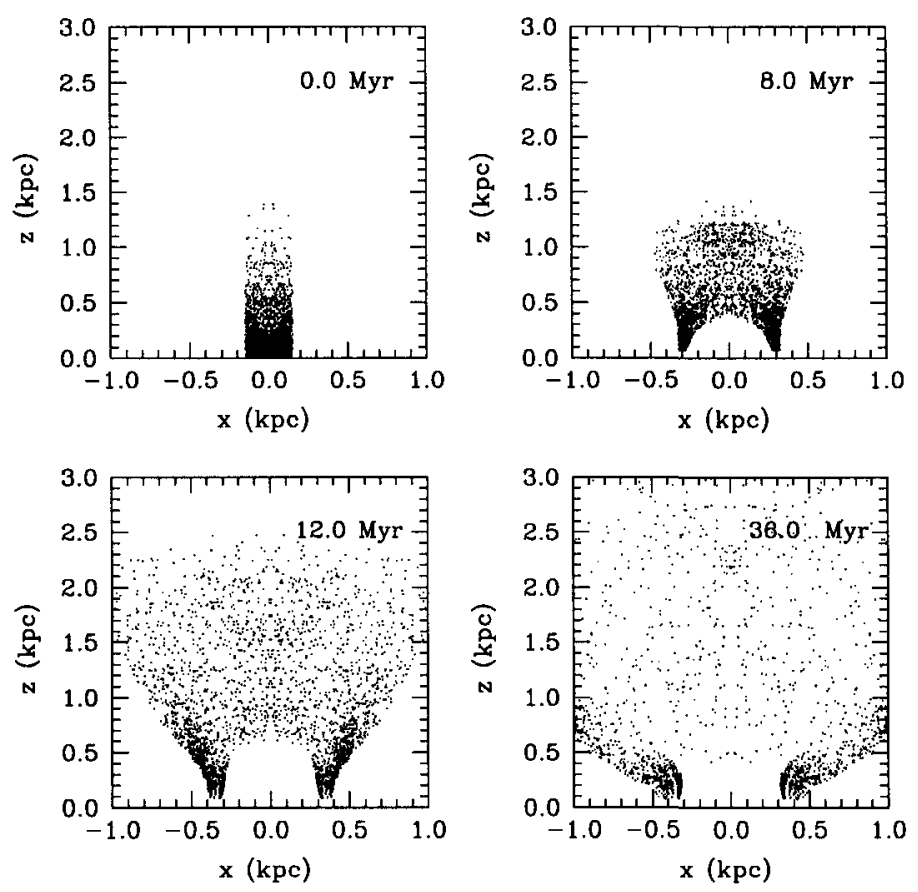

Fig. 2. Spatial distribution of dust grains for an association (located at the origin of the axes) containing $N=500 \mathrm{OB}$ stars at the evolutionary times shown in the upper corner of each panel 
Fig. 2 illustrates the spatial distribution of the dust grains (all sizes $a=$ $10-250 \mathrm{~nm}$ are shown) for a $N=500$ OB stars association at various evolutionary times. An inspection of Fig. 2 shows the presence of a central cavity completely devoid of dust swept by the very strong radiation pressure. In addition, the configuration of the region affected by the radiation field resembles now an inflated bubble in which a relevant fraction of grains has been pushed well out of the main disk of the Galaxy, at $|z|>3 \mathrm{kpc}$; it is also possible to identify the filamentary structures that form the lateral walls of the dusty chimney. A vertical cut through the origin (i.e. above the center of the association) shows that the $z$-distribution of the dust becomes much flatter with time than the original exponential one. Thus, the dust is rather homogeneously distributed by the radiation pressure in the portions of the halo above the associations; moreover, this implies that the dust-to-gas ratio has to be larger in the upper halo than in the disk. Finally, since the drag force becomes vanishingly small because the gas density is rapidly decreasing with height, some grains can continue their journey for several $\mathrm{kpc}$ before they feel the gravitational pull of the Galaxy. The velocities achieved by the grains are rather high, and they can exceed (for a $N=500 \mathrm{OB}$ stars association) $100 \mathrm{~km} \mathrm{~s}^{-1}$. Smaller grains tend to move faster since, neglecting drag forces, the ratio of the radiation-to-gravitational force is $\propto a^{-1}$.

\section{A Possible Scenario}

If dust is present in galactic halos it could be responsible for several important detectable effects. In addition to the standard direct observations discussed in the Introduction involving either the obscuration of distant object by the grains or their FIR emission, a number of indirect but nonetheless intriguing consequences of dust can be individuated and used to put constraints on any theory of galactic halos.

The simple sketch presented in Fig. 3 helps building a possible scenario which might be quite common among star-forming spiral galaxies at any epoch and includes both the effects discussed above and additional ones. The gaseous disk of the Milky Way is now understood to consist of at least two components: a neutral one, which extends roughly up to $z \sim 0.5 \mathrm{kpc}$ (including the so-called Lockman layer), and an ionized component (Reynolds layer) whose vertical extent is less clear but of the order of $1 \mathrm{kpc}$. An ordered magnetic field component with strength $|\mathbf{B}| \sim 3 \mu \mathrm{G}$ in the plane of the disk is known to be present (Heiles 1995). As we have seen above, OB associations will produce (before SN explosions) a strong ionizing flux and an HII chimney, pushing the grains into the halo by radiation pressure. Due to the presence of the magnetic field, and due to the higher density of the gas and hence enhanced drag, grains located initially at low- $z$ will be prevented from being evacuated, if they have a non-zero charge. This last point is very important, since in spite of the fact that we have not included the Lorentz 


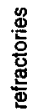
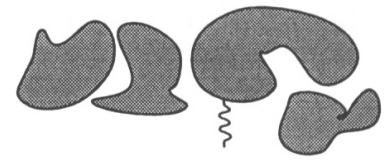

00

H II

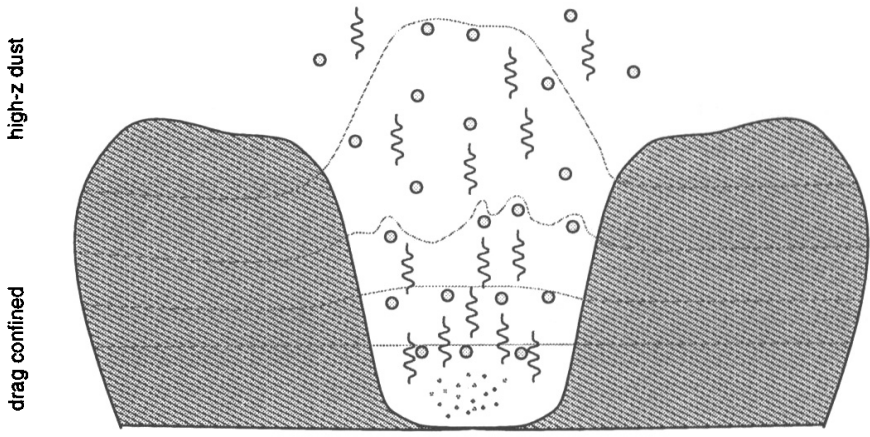

H I

Fig. 3. Schematical representation of the various processes occurring above a HII chimney discussed in the text

force in our simulations, nevertheless our accurate determination of the grain charge shows that considerable time intervals are spent by grains in a neutral charging state: this is due to the balance between photoelectric and collisional charging. Thus, at least intermittently, grains will be free to move even in presence of a magnetic field above HII chimneys. During the periods in which they are charged, grains are trapped and spiral around field lines. However, grains located at higher altitudes $(z \sim 0.3-0.4 \mathrm{kpc})$ will experience a much reduced drag force and therefore the pressure of the dust "fluid" will be felt by the magnetic field. This pressure adds up with the standard thermal and cosmic rays one and will enhance the Parker instability, thus originating a vertical component of $\mathbf{B}$. The criterion for instability when a dust pressure term $\delta=p_{r} / p_{t h}$ is added to the magnetic field, $\alpha=p_{B} / p_{t h}$, and cosmic ray, $\beta=p_{c r} / p_{t h}$ pressure (where $p_{t h}$ is the thermal pressure of the gas) becomes in the long wavelength limit:

$$
\gamma<\frac{(1+\alpha+\beta+\delta)^{2}}{1+\beta+\delta+(3 / 2) \alpha}
$$

where $\gamma=5 / 3$ is the ratio of the specific heats. Since $\delta \gg 1$ above HII chimneys, radiation pressure can profoundly modify the topology of the magnetic field. Grains spiralling along the vertical field lines can now flow into the halo and eventually be sputtered (nonthermal sputtering is likely due to the high grain velocities), releasing the refractory elements detected by absorption lines experiments through the halo and known to have a scale height of the order of a few kpc, depending on the species (Edgar \& Savage 1989; Lipman \& Pettini 1995; see Ferrara et al. 1991 for a detailed calculation). This 
implies that the size distribution of grains can deviate substantially from a MRN distribution in the halo, with an excess of small grains. Finally, collisions with the gas atoms will put grains into rotation and, being charged, they will emit radiation with a spectrum sharply peaked at a cutoff frequency typically in the range $10-100 \mathrm{GHz}$ (Ferrara \& Dettmar 1994). The estimate of the global dust mass loss due to this effect from galactic disks depends on the detailed modelling of the star formation rate in the galaxy and other galactic properties and will be given in Ferrara \& Shull (1997).

\section{Cosmological Implications of Dusty Halos}

There are several consequences of the presence of dust in the halos of galaxies and perhaps in the intergalactic medium. The most obvious one concerns the determination of the deceleration parameter, $q_{0}$; indeed, this parameter is extremely sensitive to the assumptions concerning the extinction. In an important paper, Margolis \& Schramm (1977) have estimated that the correction due to EGD to $q_{0}$ is

$$
\left(\Delta q_{0}\right)=2.8 \times 10^{3}\left(\frac{A_{v}}{1 \mathrm{mag} \mathrm{Mpc}^{-1}}\right) h^{-1},
$$

where $A_{v}$ is the visual extinction and $h=H_{0} / 100 \mathrm{~km} \mathrm{~s}^{-1} \mathrm{Mpc}^{-1}$. Thus, as little as $2 \times 10^{-4} \mathrm{mag} \mathrm{Mpc}^{-1}$ absorption would cause a measurement error of $\left(\Delta q_{0}\right) \sim 1$

Extragalactic dust (i.e. dust outside the main body of galaxies) might also affect QSO number counts (Heisler \& Ostriker 1988). The ratio of the total cross section of dusty absorbers to the total sky area in a $\Omega=1$ universe at redshift $z$ is

$$
\mathcal{F}(z)=\frac{2 \pi R(0)^{2} n(0) c}{3 H_{0}}(1+z)^{3 / 2}
$$

where $n(0)$ and $R(0)$ are the local number density and size of the absorbers. Substituting typical numbers for $\left(n(0) \sim r_{0}^{-3} \sim 0.008 h^{3} \mathrm{Mpc}^{-3}\right.$, where $r_{0} \sim 5 h^{-1} \mathrm{Mpc}$ is a correlation scale for galaxies and $R(0) \sim 100 \mathrm{kpc}$ ) halos associated with galaxies, we conclude that $\mathcal{F}(z)=1$ at redshift $(1+z)=$ $\left(2 / h^{2}\right)^{2 / 3}=1.58 h^{-1 / 3}$. Thus high redshift objects are likely to suffer substantial extinction by intervening systems. As a final remark, the release of heavy elements following grain sputtering in the halo might be relevant to the interpretation of QSO absorption lines in metal line systems.

Acknowledgements. I deeply acknowledge the constant inspiration provided by the friend Prof. Lyman Spitzer, Jr. "from whom I have learned a lot". I also thank Mike Shull, my collaborator in this project. 


\section{References}

Davies, J. I., Trewhella, M., Jones, H., Lisk, C, Madden, A. \& Moss, J. 1997, preprint

Dettmar, R.-J. 1992, Fund. Cosm. Phys., 15, 14

Dickey \& Lockman 1990, ARA\&A, 28, 215

Dove, J. B. \& Shull, J. M. 1994, ApJ, 430, 222

Draine, B. T. 1978, ApJSS, 36, 595

Draine, B. T. \& Salpeter, E. E. 1979, ApJ, 231, 77

Draine, B. T. \& Sutin, 1984, ApJ, 320, 803

Dwek, E. \& Arendt, R. G. 1992, ARA\&A, 30, 11

Edgar, R. J. \& Savage, B. D. 1989, ApJ, 340, 762

Ferrara, A, Ferrini, F. Franco, J. \& Barsella, B. 1991, ApJ, 381, 137

Ferrara, A. \& Dettmar, R.-J. 1994, ApJ, 427, 155

Ferrara, A., \& Shull, J. M. (1997), in preparation

Frisch, P. C. 1995, Sp. Sc. Rev., 72, 499

Heiles, C. 1995, in The Physics of the Interstellar Medium and Intergalactic Medium, eds. A. Ferrara et al., ASP Conf. Series, 80, 507

Heisler, J. \& Ostriker, J. P. 1988, ApJ, 332, 543

Leroy, J. L. 1993, A\&A, 274, 203

Lipman, K. \& Pettini, M. 1995, ApJ, 442, 628

Mac Low, M. M. \& Norman, M. L. 1992, BAAS, 181, 6706

Margolis, S. H. \& Schramm, D. N. 1977, ApJ, 214, 339

McKee, C. F. 1989, in Interstellar Dust, IAU Symp. 135, eds. L. J. Allamandola \& A. G. G. M. Tielens, (Kluwer: Dordrecht), 431

Miniati, F., Jones, T., Ferrara, A. \& Ryu, D. (1997), ApJ, submitted (astroph /9706208)

Norman, C. A. \& Ikeuchi S. 1989, ApJ, 345, 372

Normandeau, M, Taylor, A. R., \& Dewdney, P. E. 1996, Nature, 380, 687

Ricotti, M., Ferrara, A., \& Miniati, F. 1997, ApJ, in press, astro-ph/9702143

Seab, C. G. 1987, in Interstellar Processes, eds. D.J. Hollenbach \& H. A. Thronson Shapiro, P. R. \& Field, G. B. 1976, ApJ, 205, 762

Shull, J. M. \& Saken, J. M. 1995, ApJ, 444, 663

Sofue, Y., Wakamatsu, K. \& Malin, D. 1994, AJ, 108, 231

Tielens, A. G. G. M., McKee, C. F., Seab, C. G. \& Hollenbach, D. J. 1994, ApJ, 431,321

Tinbergen, J. 1982, A\&A, 105, 53

Tomisaka, K. 1990, ApJL, 361, 5

Zaritsky, D. 1994, AJ, 108, 1619 